\title{
CATALOGACIÓN DE DOCUMENTOS CARTOGRÁFICOS EN RDA
}

\section{Eva Novotná}

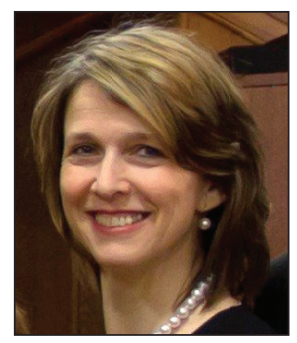

Eva Novotná es master y doctora por el Instituto de de Biblioteconomía y Estudios de Información en la Universidad Carolina (Cuni) de Praga, República Checa, y master del Programa de Formación para Profesores de Secundaria Superior en Estudios Religiosos por la Facultad de Teología Católica en la Cuni, en Praga. Ha trabajado durante varios años como directora de bibliotecas especializadas. Ha llevado a cabo varios proyectos sobre autoridades y bibliografías, como por ejemplo las Autoridades de nombres cristianos de Bohemia, y la Bibliografía geográfica checa online, con 180.000 registros y 30.000 textos completos. Desde 2011 es directora de la Colección de Mapas de la Facultad de Ciencias, Cuni, y co-directora del proyecto Tecnología para procesar las colecciones de mapas de la República Checa. En éste, se ha encargado de catalogar los documentos cartográficos, preparar metodologías de las RDA, digitalizar, metadatos y acceso a los documentos cartográficos. Es autora de artículos, exposiciones, catálogos y libros.

http://orcid.org/0000-0001-9973-2532

Geographical Library, Faculty of Science, Charles University Albertov, 6. 12843 Praha 2, República Checa eva.novotna@natur.cuni.cz

\section{Resumen}

Se muestran las reglas de catalogación RDA (Resource description and access) que parten del modelo conceptual de FRBR y FRAD, describiendo las entidades básicas como la obra, su expresión, realización, unidad, atributos y relaciones o contexto. Se explica la estructura de RDA y los cambios fundamentales de las reglas existentes AACR, con respecto a la nueva terminología y modelo de catalogación, tratándose en concreto de la regla de tres autores, supresión de siglas, anotaciones de números y de errores y uso de corchetes. Se informa sobre los nuevos campos de MARC 21 para los datos editoriales 264 así como sobre el campo 3XX para el tipo de contenido, medio y portador de datos. Nos centramos en el problema de cómo aplicar estas modificaciones en los documentos cartográficos, especialmente en lo que se refiere a los campos de datos matemáticos 255 destinados a escalas, visualizaciones cartográficas y coordenadas, así como los determinados para la descripción física, citando posiblidades de uso. Un papel fundamental en el desarrollo de las RDA y formación de catalogadores lo desempeña el grupo especializado en documentos cartográficos Magirt (ALA). Las reglas son lo suficientemente flexibles como para que puedan servir tanto a catalogadores como a agencias que se dedican a la catalogación, por lo cual será necesario interpretarlas en función de las costumbres que tiene cada país en su sistema de catalogación. Las RDA ya han sido traducida a las lenguas más importantes del mundo.

\section{Palabras clave}

Functional requirements for bibliographic records, FRBR, Resource description and access, RDA, Anglo-American cataloguing rules (AACR2), Catalogación, Documentos cartográficos, Mapas, Estándares, Normas, Reglas, Descripción, Metadatos, Ejemplos.

\section{Title: Cataloging cartographic documents with RDA}

\begin{abstract}
The article discusses new RDA (Resource description and access) cataloguing rules, which are based on the FRBR and FRAD conceptual model. The basic entities (work, expression, manifestation and item), as well as their attributes and relationships are described, and RDA structure and substantial changes from existing AACR2 rules explained. New terminology, new cataloguing models, and specifically "the rule of three" are included, no abbreviations, annotations of numbers and errors, and use of square brackets. New MARC 21 fields are explained: the 264 field for publisher data and the 3XX field for content, media and carrier types. The paper addresses the application of these changes in cartographic documents, especially in the 255 field for mathematical data elements, containing a sub-field for scale, cartographic projections and coordinates, and in the physical description field, including record samples. The essential role in the RDA development and training of cataloguers for cartographic documents is carried out by an expert group called Magirt (ALA). The rules offer freedom of application for cataloguers and cataloguing agencies; therefore, their interpretation is required, taking into account national cataloguing conventions. The instructions have already been translated into the major world languages.
\end{abstract}




\section{Keywords}

Functional requirements for bibliographic records, FRBR, Resource description and access, RDA, Anglo-American cataloguing rules (AACR2), Cataloging, Cartographic documents, Maps, Standards, Rules, Document description, Metadata, Examples.

Novotná, Eva (2014). “Catalogación de documentos cartográficos en RDA“. El profesional de la información, marzo-abril, v. 23, n. 2, pp. 195-203.

http://dx.doi.org/10.3145/epi.2014.mar.13

\section{Introducción}

Las instrucciones RDA (Resource description and access) creadas por el Joint Steering Committee (JSC) for AngloAmerican Cataloguing Rules, son una nueva y unificada normativa de catalogación, que, a su vez, reemplazan a las AACR2 usadas en la actualidad (Anglo-American Cataloguing Rules, 2010). Entre las organizaciones participantes en el JSC están, entre otras: American Library Association (ALA), Australian Committee on Cataloguing (ACOC), British Library (BL), Canadian Committee on Cataloguing (CCC), Chartered Institute of Library and Information Professionals (Cilip) y Library of Congress (LC).

Una vez transcurrido el período de prueba, las reglas RDA fueron implementadas en marzo de 2013 en la Library of Congress, así como en las bibliotecas nacionales de Canadá, Australia, Gran Bretaña y Alemania. Además, se están llevando a cabo de forma gradual cambios en los registros contenidos en el catálogo mundial OCLC (Online Computer Library Center) (Whitacre; Fujikawa, 2013). En el actual ambiente de catalogación compartida es muy probable que también catalogadores de otros países empiecen a crear registros con las $\mathrm{RDA}^{1}$, ya que las mismas han sido traducidas ya al chino, francés, alemán y español (ALA, 2010).

El presente artículo se basa en los Requisitos funcionales de los registros bibliográficos (FRBR) que desarrollan el modelo conceptual de entidades, atributos y relaciones, de los cuales parten las nuevas instrucciones de catalogación (Celbová, 2001). La obra en cuestión ha sido publicada en forma impresa en 2010 (ALA, 2010), y desde entonces está siendo sometida a constantes revisiones. ALA publica en la web RDA Toolkit (2013) tanto las reglas como sus actualizaciones y ejemplos de cómo usarlas. Otra fuente importante de información sobre RDA es el propio JSC (2013). Las autoras Anne Welsh y Sue Batley publicaron en 2012 el libro Practical cataloguing: AACR, RDA and MARC 21 (Welsh; Batley, 2012), en el cual acercan al usuario la filosofía básica de la catalogación, los estándares catalográficos, así como los procesos FRBR, citando múltiples ejemplos de catalogación tanto en AACR como RDA, e incluyendo la portada de cada uno de los documentos descritos.

Desgraciadamente, la información relativa a documentos cartográficos es muy escasa. Los materiales citados a continuación, procedentes de cursos de formación, han sido de gran utilidad:

- Back to the basics. An Alcts pre-conference presentado en la conferencia ALA de 2013, por la Association for Library Collections \& Technical Services, en Chicago.

- Chris Oliver en su presentación Understanding RDA: a guide to some basic concepts explicó de forma clara y concisa las bases de FRBR y FRAD, que representan el modelo conceptual del cual parte RDA (2013b).

- Otro trabajo del mismo autor RDA: Highlights of desciptive changes está dedicado a cambios emprendidos en las descripciones RDA (Oliver, 2013).

- Luiz Mendes impartió una ponencia acerca de los cambios que se están operando en los puntos de acceso (2013).

- Mucho interés suscitó también la exposición View from the utilities a cargo de las autoras Cynthia Whitacre y Georgia Fujikawa (2013) cuyos objetivos fueron los intercambios y las posibilidades de colaboración entre bibliotecas, así como el planning de actualizaciones de RDA a realizar en el catálogo de $O C L C$.

Para este artículo la autora ha utilizado también materiales recogidos en cursos de formación y sesiones de trabajo:

- Del grupo ALA sobre el tratamiento de documentos cartográficos Magirt (Map and Geospatial Information Round Table) (2013), cuyos integrantes elaboran métodos de catalogación de documentos cartográficos en RDA.

- Webinarios que ALA ofrece con regularidad, dedicados tanto a los postulados básicos de RDA, como a tipos de documentos específicos.

- Webinario de Paige G. Andrew RDA and cartographic materials: mapping y new route (2011), dedicado a los cambios generales que han experimentado las reglas, uso de nuevos campos 3XX e impacto que dichos procesos han tenido sobre la catalogación de documentos cartográficos.

- El mismo autor, junto con Susan Moore, organizó en el marco de la conferencia en Chicago, otro curso formativo denominado Maps the RDA way (Andrew; Moore, 2013), en el cual desarrollaba más a fondo su ponencia dada en 2011, centrándose en primer lugar en los cambios de RDA en documentos cartográficos. Describieron los nuevos campos brindando múltiples ejemplos y ejercicios concretos sobre la materia.

- Otro webinario, además de responder a las preguntas planteadas por los participantes del seminario, fue enfocado a cuestiones específicas, como la del uso del campo 264, catalogación de fotocopias, aplicación de corchetes y posibilidades de los signos de puntuación ISBD (Andrew; Moore, 2013).

- Ponencia dedicada especialmente a los documentos cartográficos a cargo de Katherine Rankin y Mary-Lynette Larsgaard (2012).

La autora expresa un especial agradecimiento a Susan Moore por haber respondido por vía mail de forma abnegada a todas sus preguntas. 


\section{Adaptación del catálogo al sistema FRBR}

La estructura teórica de RDA parte de los modelos conceptuales FRBR (Functional requirements for bibliographic records, 1998, 2001) y FRAD (Functional requirements for authority data, 2009). Ambos modelos se desarrollaron bajo los auspicios de IFLA (International Federation of Library Associations and Institutions) (Oliver, 2013). Tales modelos se basan en las relaciones entre entidades y analizan los datos bibliográficos y los de autoridad desde el punto de vista del usuario, es decir en función de la forma en que serán utilizados. Mientras que las reglas AACR2 estaban ordenadas en función de los tipos de documentos, RDA no hace distinción entre ellos, tomando en consideración únicamente el hecho de si la entidad a catalogar es una obra (work), expresión (expression), manifestación (manifestation) o ítem (item). Los FRBR fueron publicados por primera vez en 1998 (Celbová, 2001). Examinan la concepción intelectual de la obra, el concepto del libro así como las relaciones de cara a las copias de la obra que están en otras bibliotecas. Las relaciones son lo que debe representar el componente fundamental de cada catálogo. El cometido del catalogador no consiste sólo en describir la obra que está en sus manos, sino también en registrar las relaciones que la misma tiene dentro del universo bibliográfico. Su trabajo no termina con anotar el nombre del autor y el año en que fue publicado el libro; además de ello debe apuntar todos los datos que pudieran resultar de utilidad para el usuario final. Los FRBR dan testimonio del hecho de que cada objeto tiene forma, contenido y complejas relaciones de las distintas versiones entre sí (Wels; Batley, 2012).

Los requisitos funcionales básicos para registros bibliográficos se definen según las necesidades que tiene el usuario. Éste quiere: 1. Encontrar una entidad (investigación), 2. Identificar una entidad (pertinencia), 3. Seleccionar una entidad (búsqueda), 4. Acceder (obtener el texto completo online o pedirlo prestado).

Los requerimientos básicos del modelo FRAD son similares: 1. Encontrar una entidad, 2. Identificarla, 3. Explicar relaciones o contexto, 4. Autorizar (comprender) (Oliver, 2013).

\subsection{Elementos básicos del modelo: entidades, atribu- tos y relaciones}

Las entidades bibliográficas se dividen en tres grupos:
1. Creaciones fruto de una actividad intelectual o artística: obra, expresión, manifestación, ítem. Para las cuatro entidades se usa también la sigla WEMI (Rankin; Larsgaard, 2012).

2. Entidades que responden por el contenido intelectual y artístico, producción, distribución o administración: persona (person), corporación (corporate body).

3. Entidades complementarias como objetos de una actividad intelectual o artística: concepto (concept), objeto (object), evento (event), lugar (place) (Celbová, 2001).

Las relaciones primarias dentro del primer grupo se dividen en tres:

a. obra realizada mediante

b. expresión materializada en

c. manifestación ejemplificada por un ítem (Wels; Batley, 2012).

Como ejemplo del primer grupo nos podemos servir del conocido atlas de Abraham Ortelius Theatrvm orbis terrarvm (1584). La obra como idea fue realizada por medio de la expresión del texto original en neederlandés, y fue escrito y materializado en la manifestación publicada por el editor Abraham Ortelius en Amberes, en 1584. El ejemplar se encuentra en la colección de mapas de la Facultad de Ciencias Naturales de la Univerzita Karlova, código 3233299719.

\subsection{Entidades del segundo y tercer grupo}

La responsabilidad del contenido intelectual y artístico, la de la producción física, y la de la divulgación o administración de entidades del primer grupo se expresan con las entidades del segundo. Se trata de personas (creadores, cartógrafos, editores, etc.), familias (propietarios, narradores, etc.) y corporaciones (editores, editores literarios, etc.) que además pueden ser objeto de la obra. 
Las relaciones de responsabilidad están vinculadas con el primer grupo, es decir, con la obra creada por personas, familias y corporaciones, con la expresión que realizan, con la manifestación que producen, y finalmente con el ítem que poseen.

Los objetos de la obra vienen expresados en el tercer grupo en forma de concepto, objeto, acción y lugar. El concepto es una idea abstracta que designa el objetivo de la obra. Cada obra puede tener varios conceptos. El objeto es el objeto material de la obra, por ejemplo un teodolito o una torre de observación (Oliver, 2013).

Tabla 1. Registro bibliográfico en formato MARC y FRBR para documentos cartográficos (según Steven J. Miller) (Rankin; Larsgaard, 2012).

$\begin{array}{ll}1 \mathrm{XX} / 240 & =\text { obra, expresión } \\ 245-260,490 & =\text { manifestación } \\ 255 \$ \mathrm{a} & =\text { expresión, \$b = obra, \$c = obra (Moore, 2013) } \\ 300 & =\text { expresión, manifestación } \\ \text { otro 33X } & =\text { obra, expresión, manifestación } \\ 5 \mathrm{XX} & =\text { obra, expresión, manifestación } \\ 700-730 & =\text { obra, expresión } \\ 760-787 & =\text { obra, expresión, manifestación } \\ 8 \mathrm{XX} & =\text { obra, expresión y manifestación }\end{array}$

\subsection{Atributos}

Los usuarios utilizan atributos para encontrar determinadas entidades que bien forman parte de la entidad en cuestión, o bien se pueden obtener desde fuera. Por ejemplo, en la entidad de obra registramos nombre, forma, coordenadas, equinoccio, etc. En la entidad de expresión registramos forma, idioma, escala, proyección cartográfica, técnica de visualización, interpretación del relieve, mediciones geodésicas, verticales y de coordenadas, etc. Entre los atributos de la entidad de manifestación cuentan los datos referentes a la responsabilidad, edición, lugar de publicación, forma del portador etc. Atributos del ítem: identificador de ítem (código de barras), su origen y estado, restricción del acceso al ítem, etc. (Celbová, 2001). En el caso de persona, los atributos del segundo grupo engloban, entre otros, nombre, fechas, título y otras designaciones de la persona. En cuanto a la corporación, los atributos, pueden ser lugar, fecha, número, historia de la corporación. Los atributos de familia abarcan el tipo, fecha, lugar relacionado con la misma, historia, etc. (Oliver, 2013).

\subsection{Relaciones}

Las relaciones principales se establecen entre las entidades básicas: obra, expresión, manifestación e ítem. Las relaciones secundarias se llevan a cabo entre:

1. Persona, familia o corporación y fuente

2. Una fuente y otra

3. Persona, familia o corporación y otra persona, familia o corporación.

\section{Organización y estructura de RDA}

FRBR y FRAD se reflejan en RDA. Las reglas se dividen en dos secciones principales: las secciones 1 a 4 describen atributos de cada una de las entidades, y las 5 a 10 explican las relaciones existentes entre las mismas.
En los anexos se encuentra información acerca del uso de mayúsculas, abreviaturas, artículos iniciales, sintaxis para datos descriptivos así como los selectivos, informaciones complementarias sobre nombres de personas, títulos nobiliarios, rangos, fechas del calendario cristiano, designadores de relaciones entre entidades, vocabulario, registro ( $A L A$, 2010).

\subsection{Cambios sustanciales con respecto a AACR2}

Las RDA trabajan con nueva terminología para encabezamiento (access point), datos de responsabilidad (creator), responsabilidad física, es decir, medio para almacenar la información (carrier). Los elementos básicos (core elements) se describen en el capítulo 0.6 y en la web de la Library of Congress (2012). La abreviatura GMD (general material designation) designa cambios realizados en la clasificación general del tipo de documento que ha sido sustituido por 3 elementos independientes: tipo de medio, tipo de portador y tipo de contenido. En lugar de usar una denominación unificada se emplea el "título preferido" de la obra (ALA, 2013). En vez de hablar de encabezamiento principal y secundario, se usa título preferido y punto de acceso autorizado para creador (access point for creator). Referencias tipo "véase" serán sustituidas por una variante de elemento de acceso; véase también elemento de acceso autorizado para entidades relacionadas (Kuhagen, 2010).

Las reglas hacen posible que tanto la interpretación como el uso sean relativamente libres, dependiendo su empleo de la decisión del catalogador, y de la institución o política nacional de catalogación (Oliver, 2013). Las bibliotecas norteamericanas disponen de una plataforma establecida en la Library of Congress (Library of Congress - Program for cooperative cataloging policy statements, LC-Pccps), donde se publican interpretaciones nacionales, correcciones, complementos, enmiendas así como opiniones para catalogación en $\operatorname{RDA}(A L A, 2013)$.

Aclarando las informaciones poco inteligibles u ocultas, RDA ayuda al usuario a identificar con mayor facilidad documentos de su interés. Los registros han cambiado de forma sustancial en lo que se refiere a la reglas de tres autores, abreviaturas, números, errores, incorrecciones y corchetes.

Los números romanos, que aparecen ante todo en los datos referidos a publicaciones y números de edición, se han transformado en arábigos. En RDA los números romanos se registran tal y como lo prefiera la agencia catalogadora o según la fuente de descripción (1.8.2). Las incorrecciones, errores tipográficos y demás fallos se tienen que registrar en la misma forma en que aparecen en el original, sin incluir la nota sic o i.e., citando en el campo 246 [eventualmente en el campo reservado para notas (Moore, 2013) ${ }^{2}$ ] la denominación correcta (2.3.1.4.). Los corchetes se pueden usar únicamente para los datos procedentes de otra fuente. Cada uno de los datos se ha de citar dentro de un paréntesis diferente, no es posible juntar unos con otros (Rankin; Larsgaard, 2012).

Aparte del campo 264 (Library of Congress, 2011) destinado a datos editoriales, es nuevo también el campo para portador 33X. Así mismo ha cambiado la relación creador - signos 
de puntuación. La máxima más importante para la nueva catalogación es: "Regístralo tal como lo ves" (Kuhagen, 2010).

Los signos de puntuación son opcionales porque en RDA no están sujetos a ningún tipo de reglas concretas. Las RDA tienen que ser flexibles y de fácil divulgación, para poder conformarse sin esfuerzo según diferentes formatos de metadatos (la puntuación dificultaría la conversión a los diferentes formatos de metadatos). Por otro lado, se puede seguir usando la ISBD (International standard bibliographic description). Se está viendo la posibilidad de cambiar el formato de intercambio MARC21, lo cual conllevaría cambios en los signos de puntuación (Andrew, 2011).

\subsection{Datos referidos a responsabilidad y tres autores}

Los datos referidos a responsabilidades se registran en la misma forma en que aparecen en la fuente de la descripción. Es posible omitir informaciones siempre y cuando no se pierda información importante. Desde hace poco, en RDA cabe la posibilidad (2.4.1.4) de citar los títulos y afiliaciones de los autores o se puede prescindir de ellos. Ya no se indican códigos referentes al tipo de autoría, sin embargo, lo que se detalla, es la responsabilidad. Ejemplo:

1001 \$a Kreibich, František Jakub Jindřich \$d 1759-1833, \$e cartógrafo

Ejemplo del uso de títulos y rangos:

$24500 \$ a C a r t e$ de la Turquie d'Europe \$h [documento cartográfi$\mathrm{co}$ / \$ $\$$ nouvellement dressée et desinée par Al. S Konrad capitaine d'artillerie pensionné de S. M. J. \& R. Ap. ; d'aprés G. Palma J. Riedl et et; chez Artaria et Compagnie editeurs proprietaires; sur la nouvelle édition de la carte du géneral F. G. Vaudoncourt

Según la instrucción principal (2.4.1.5.), aparte de la posibilidad de registrar más de tres autores, existe la posibilidad de registrar el primero y entre corchetes anotar el total de los mismos [y el total de los demás autores].

Ejemplo:

AACR2 = Europa [documento cartográfico] / bearbeiten nach F. de Beaumont ... [et al.].

RDA = Europa [documento cartográfico] / bearbeitet nach F. de Beaumont, Berghaus, Bory St. Vincent, Boué, Brongniart, v. Buch, Buckland, Charpentier, Dechen, Eichwald, Ferd, Müller, Geenough, Grimm, Griesenbach, Hiesinger, v. Holf, Hoffmann, v. Humboldt, Klöden, König, Koch, v. Leonhard, v. Lilienstern, Mendelssohn, Merleker, Ober-Müller, Pusch, Reichard, Ritter, Roch, de Rougemont, Stieler, M. Wagner, Zeune u. a.

$\mathrm{O}$

RDA = Europa [documento cartográfico] / bearbeitet nach F. de Beaumont [y otros 34].

En RDA no existe limitación en lo que se refiere al número de autores (6.27.1.3.). En el caso de que haya más personas, familias o corporaciones responsables por la creación de la obra, se constituyen datos selectivos (the authorized access point) en el siguiente orden: a. dato selectivo referido a persona, familia o corporación, b. Título preferido de la obra.

\subsection{Abreviaturas}

En RDA se eliminan abreviaturas de uso común en el proceso de catalogación, por ejemplo las usadas para el caso de autoridad (d., fl., ca, cent.), series (ed. rev.), datos matemáticos cartográficos (ca, approx.), datos físicos (p., v., ill., col.), ediciones literarias (vol., bd.) notas. El símbolo correspondiente a $\mathrm{cm}$ se sigue utilizando, ya que en el caso de símbolos métricos no se trata de abreviaturas. En lo que se refiere a escalas, se puede indicar una escala verbal, usando los siguientes símbolos (7.25.5.3).

Basándose en la regla 3.4.5.3. las páginas que no llevan numeración se enumeran sólo aproximadamente (approximately) estimando su total con una cifra igualmente aproximada. No se usa la abreviatura "aprox." ni corchetes.

Por ejemplo:

93 páginas sin numeración (no poner: [93] p.), aproximadamente 500 páginas (no poner aprox. 500 p.).

También es posible indicar que se trata de un volumen cuyas hojas no están numeradas, por ejemplo: 1 volumen (con páginas sin numeración).

Ejemplos:

300 \$a 84 páginas sin numeración, 68 páginas: \$b ilustraciones ; \$c $27 \mathrm{~cm}$

Los datos relativos a la edición de la obra se registran en la forma en que están anotados en la fuente, es decir, no es obligatorio abreviarlos, pero tampoco hay que registrarlos en toda su extensión (2.5).

Ejemplo:

$\begin{array}{ll}\text { Fuente } & =\text { Tercera edición revisada } \\ \text { AACR2 } & =3^{\text {a }} \text { ed } \text { rev. } \\ \text { RDA } & =\text { Tercera edición revisada }\end{array}$

No se usan abreviaturas en latín. En el caso de que no se pueda averiguar ni de manera aproximada el lugar de publicación del documento, no se pone la abreviatura latina de [s.l.] (sine loco), sin indicar este hecho entre corchetes independientes [lugar de publicación no identificado] (place of production not identified).

Análogamente, si en el documento no viene indicado el nombre del productor (producers' name), y tampoco es posible averiguarlo de otras fuentes, no se pone la abreviatura latina [s.n.] (sine nomine), sino el término [productor no identificado] [producer not identified]. Cada una de las 
frases hay que introducirla entre corchetes independientes (2.7.4.7).

\subsection{Nuevo campo 264 para datos de publicación}

Según las instrucciones RDA los datos relativos al productor se registran de la misma forma en que aparecen en la fuente descrita (2.8.2). Los cambios sustanciales se pueden resumir en tres puntos:

- El lugar de publicación se registra con la jurisdicción más amplia, si existe más de un lugar de publicación, hay que reseñarlos todos. En el caso de que no sea posible averiguar el lugar de publicación, hay que anotar entre corchetes: [Lugar de publicación no identificado] (Wels; Batley, 2012).

- La regla 2.7.2.6 solventa el problema de si no aparece en la fuente el lugar de publicación. En este caso hay que anotar entre corchetes un lugar posible donde la obra pudiera haber sido publicada.

Antes, en AACR2 el nombre del productor se registraba en la forma más corta posible, mientras que ahora en RDA se recomienda transcribir su nombre en la misma forma en que aparece en la fuente descrita (2.7.4).

La máxima más importante para la nueva catalogación es: "Regístralo tal como lo ves"

El subcampo \$c destinado a fechas, ha sufrido cambios. Las fechas aproximadas o probables se indican así: [1969?] (se deja de usar la abreviatura „aprox.“). Los intervalos de años se tienen que registrar igualmente con un signo de interrogación: [entre AAAA y AAAA?], así como decenios y siglos [entre 1970 y 1979?] o [entre 1800 y 1899]. Una novedad es el registro para la fecha más tardía posible [no antes/ después de 28/10/1918] (1.9.2.). El derecho de autor o copyright se puede registrar bien entero o con el símbolo.

Ejemplos:

2641 \$c [no antes/después de 1938]

2641 \$c [fecha de publicación no identificada], (02002 [copyright

2002] (Rankin; Larsgaard, 2012)

\section{Documentos cartográficos en RDA}

La gran mayoría de instrucciones RDA relativas a documentos cartográficos, se encuentra en los capítulos 2, 3, 6 a 7 (Andrew, 2011).

Los puntos de acceso para documentos cartográficos son: escala, coordenadas, tipo de portador, extensión del documento cartográfico, dimensiones del mapa. La proyección cartográfica es opcional.

\subsection{Cambios en los registros de materiales cartográ- ficos en el campo 255 MARC 21}

Más arriba se indicaron los cambios en el uso de abreviaturas y corchetes. A continuación se verá en qué forma éstos se utilizan en materiales cartográficos. Las instrucciones re- feridas al campo 255 de datos matemáticos (subcampo \$a, $\$ b, \$ c$ ) se encuentran en el capítulo 7 , ya que se engloban en las entidades que expresan relación con el contenido.

La escala (7.25) se expresa de forma parecida a la descrita en el capítulo 3 de las AACR2, salvo algunas excepciones. La escala se puede obtener de cualquier fuente y de varias maneras (7.25.1.2), y por este mismo motivo ya no se indica entre corchetes. En el caso de que la escala no venga citada en el documento primario hay que obtenerla, bien calculándola a partir de la escala gráfica, bien mediante un indicador natural de escala (Moore, 2012) ${ }^{3}$, y tampoco se pone entre corchetes. La escala, en cualquiera de sus formas (numérica, gráfica, descrita) siempre se expresa en forma de fracción $1: n$. Aunque no esté explícitamente indicado en ninguna parte, en las bibliotecas norteamericanas se seguirá anteponiendo al subcampo \$a la palabra Escala, exceptuando aquellos casos donde en el mapa esté explícitamente indicado, que el mapa en cuestión había sido confeccionado sin basarse en una escala. En ese caso, habrá que anotar la frase Mapa trazado sin usar escala (Andrew; Moore, 2013). Si en el mismo documento aparecen mapas con tres y más escalas, según AACR2 hay que anotar Diferentes escalas. En las RDA es posible indicar todas las escalas del mapa, registrando cada escala por separado. (7.25.1.4). Si en varios mapas integrados en el mismo documento no se sabe la escala, hay que anotar Diferentes escalas. Si alguna de las escalas se puede descifrar, hay que registrarla.

Por ejemplo:

Escala del tercer y cuarto mapa 1:250 $000(A L A, 2013)$

Un ejemplo del cambio en la forma de registrar una escala aproximada procedente del indicador natural de escala:

AACR2 = 255 \$a Escala [aprox. 1:90 000]

$\mathrm{RDA}=255 \$$ a Escala aproximada 1:90 000

Otro ejemplo de cambios: la escala se ha calculado a partir de otras unidades de medición. Como el autor del cómputo es un humano, se supone que lo hace con exactitud, por lo tanto, no hace falta usar el término "aproximado".

AACR2 = 255 \$a Escala [aprox. 1:250 000]. 9,7 cm = 5 Common leauges of Flanders. $10,3 \mathrm{~cm}=6$ Common leauges of France. $11,2 \mathrm{~cm}=$ 15 British computed miles

RDA = 255 \$a Escala 1:250 000. 9,7 cm = 5 Common leauges of Flanders. $10,3 \mathrm{~cm}=6$ Common leauges of France. $11,2 \mathrm{~cm}=15$ British computed miles

\subsubsection{Proyección cartográfica}

Los datos relativos a la proyección se pueden obtener no solamente del documento primario, sino también de cualquier otra fuente (7.26.1.1). En proyecciones no se usan abreviaturas. Por ejemplo: 255 \$b Proyección equidistante. 


\subsubsection{Coordenadas}

Según la instrucción 3.3D1 era posible indicar las coordenadas por medio de grados, minutos y segundos. A parte del registro clásico, las nuevas reglas ofrecen la posibilidad de indicar coordenadas en grados decimales (7.4.2.3) (Andrew; Moore, 2013) ${ }^{4}$. Las informaciones se pueden adaptar de cualquiera de las fuentes disponibles.

Ejemplo de cómo usar el campo 255 entero:

255 \$a Escala 1:600 000 ; \$b proyección de Gauss \$c $\left(022^{\circ} 06^{\prime} 16^{\prime \prime}\right.$

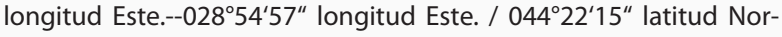
te.--041 $06^{\prime} 44^{\prime \prime}$ latitud Norte)

\subsection{Campo de datos físicos 300 MARC 21}

Para fuentes impresas, manuscritos, obras gráficas o fuentes tridimensionales cuyo contenido es de carácter cartográfico se aplican las reglas RDA 3.4.2.2-3.4.2.5. Para el caso de fuentes con contenidos cartográficos grabados en otros medios (por ejemplo, microfilm) son de aplicar las instrucciones de la regla 3.4.1. Para la descripción física del documento cartográfico se usa el número de unidades y términos detallados en la lista, sea en singular, sea en plural. Lista de términos: atlas, diagrama, globo, mapa, modelo, perfil, imagen obtenida vía satélite, sección, vista (3.4.2.2).

Como ya sabemos, RDA elimina abreviaturas, lo cual se verá reflejado en la descripción física de los documentos cartográficos. Ejemplos:

AACR2 = 300 \$a 1 mapa \$b col. ; \$c $67 \times 48 \mathrm{~cm}$ en hoja $68 \times 49 \mathrm{~cm}$ doblado en $23 \times 12 \mathrm{~cm}$

$\mathrm{RDA}=300$ \$a 1 mapa: \$b en color ; $\$ \mathrm{c} 67 \times 48 \mathrm{~cm}$ en hoja $68 \times 49 \mathrm{~cm}$, doblado en $23 \times 12 \mathrm{~cm}$

AACR2 = 300 \$a 1 atlas (89 p.): |b en color ; $\mid c 45 \mathrm{~cm}$

RDA = 300 \$a1 atlas (89 páginas): |b en color ; |c $45 \mathrm{~cm}$

Un caso a parte, es la catalogación de una obra que se describe como un conjunto. No se dispone de información sobre la cantidad total de hojas de mapas, tampoco de la ordenación de las misma. Se han conservado sólo fragmentos de la obra. En este caso el registro se hará de la siguiente forma:

300 \$a 1 mapa sobre _ hojas

La cantidad de hojas se dejará sin anotación hasta que se llegue a descubrir cuántas hojas realmente integran la totalidad de la obra (3.4.1.10) (Moore, 2013).

La instrucción 3.11.2 habla de otras características físicas como la organización de las imágenes cartográficas. Para fuentes que no sean atlas se describen las dos caras, siendo la escala de la imagen igual en la hoja/hojas.
Ejemplos:

300 \$b un mapa manuscrito en ambas caras de una hoja 300 \$b 3 mapas impresos en las dos caras de una hoja

300 \$b el mismo mapa impreso en las dos caras de una hoja, cada una de ellos en un idioma diferente.

Entre otras cosas, RDA presta atención también al registro del material físico, cuyos tipos vienen enumerados (3.6), montaje (3.8), método de producción (3.9) o polaridad y relaciones entre colores y sus matices (3.14) (Andrew; Moore, 2013).

Los signos de puntuación ISBD siguen vigentes. Igualmente se sigue trabajando con el formato MARC 21, al cual se le han añadido dos campos nuevos: 3XX y 264

\subsection{Nuevos campos $33 \mathrm{X}$}

Para las fuentes cartográficas es importante que existan nuevos campos $33 \mathrm{X}$ que se pueden repetir y que incluyan informaciones procedentes de AACR2 del subcampo 245 \$ y del campo 300.

336: tipo de contenido (RDA 6.9, obligatorio)

337: tipo de medio (RDA 3.2)

338: tipo de portador (RDA 3.3, obligatorio)

En general, podemos afirmar que el tipo de contenido responde a la pregunta "¿Qué es?", el tipo de medio a la de "¿Cómo está almacenado?" y por último, el tipo de portador a la de "¿Dónde está almacenado?" (Andrew; Moore, 2013). El contenido y portador son elementos obligatorios, por lo tanto tienen que estar registrados. Por otro lado, el tipo de medio es optativo, ya que en ocasiones sí se puede usar, mientras que en otras queda poco claro. Paige Andrew en el marco de su presentación había preparado unas tablas sinópticas, provistas de códigos para documentos cartográficos de cada uno de los campos 3XX basándose en la web de RDA en MARC (2011).

Ejemplo del campo 33X para mapa en una hoja:

336 imagen cartográfica \$b cri \$2 rdacontenido

337 no mediado \$b c \$2 rdamedios

338 hoja \$b nb \$2 rdaportador

Ejemplo de campos 33X para mapas en relieve:

336 forma cartográfica tridimensional \$b crf \$2 rdacontenido 337 no mediado $\$$ b n $\$ 2$ rdamedios

338 objeto \$b nr \$2 rdaportador 
Ejemplo de los campos 33X para globos terráqueos: (Andrew; Moore, 2013).

336 forma cartográfica tridimensional $\$ b$ crf $\$ 2$ rdacontenido 337 no mediado $\$$ b n $\$ 2$ rdamedios

338 objeto $\$ b$ nr $\$ 2$ rdaportador

Ejemplo de los campos 33X para imágenes digitales: (Andrew; Moore, 2013).

336 imagen cartográfica \$b cri \$2 rdacontenido

337 computador \$b c $\$ 2$ rdamedios

338 recurso en líne $\$ b$ cr $\$ 2$ rdaportador

\section{Conclusiones}

A medida que las nuevas reglas de catalogación se aplican en la práctica se van perfeccionando, pues cada vez existen más ejemplos de cómo usarlas; y será con su uso diario cuando se verán sus ventajas e inconvenientes. Los signos de puntuación ISBD siguen vigentes. Igualmente se sigue trabajando con el formato MARC 21, al cual se le han añadido dos campos nuevos: 3XX y 264, aunque cada vez se habla más de introducir en él limitaciones e incluso de la posibilidad de sustituirlo por otro formato. Las demás novedades en el sistema de catalogación van encaminadas a que el almacenamiento y la búsqueda de información sean más fáciles. En algunos campos aumenta el volumen de la descripción, lo cual puede implicar que el período de tiempo necesario para realizar los registros bibliográficos sea más prolongado, ante todo en bibliotecas especializadas donde difícilmente cabe esperar que los documentos que tienen en su propiedad sean descritos también en otras instituciones.

La fuerza motriz del desarrollo y divulgación de la catalogación de documentos cartográficos en RDA es el grupo de trabajo Magirt. Mary-Lynette Laarsgard y Paige G. Andrew están preparando una monografía dedicada a RDA y fuentes cartográficas. Estaba programada para el año 2013, pero al final se tuvo que posponer para 2014. Esta obra se centrará principalmente en las diferencias entre RDA y AACR2. Otra fuente importante para la catalogación de documentos cartográficos en RDA será la metodología que está preparando Tammy Wong de la Library of Congress. Estos documentos facilitarán el uso de las RDA en colecciones de mapas.

La Library of Congress ofrece un sinnúmero de materiales formativos y de tests. Las páginas de RDA Toolkit brindan regularmente webinarios tanto para principiantes como avanzados. Las reglas están siendo traducidas a varios idiomas. Muchas bibliotecas nacionales van preparando sus propias interpretaciones y registros mínimos en RDA. Todo ese proceso podría ayudar a que el cambio transcurra de forma fluida, divulgándose las nuevas reglas de catalogación con la mayor rapidez posible.

\section{Notas}

1. Identificación del registro v RDA: campo 040 contiene \$e rda

2. El uso del campo correspondiente a nota depende de la posibilidad de generar la misma por medio del indicador 1 . En el caso de que el sistema no lo permita, habrá que usar el indicador 3 y campo 500.

3. Natural scale indicator. Si los mapas contienen una escala gráfica o red cartográfica, la escala se puede deducir de ahí.

4. Para convertir minutos al sistema decimal hay que dividirlos por 60 . Por ej., $122^{\circ} 45^{\prime}$ equivale a 122,75 grados.

\section{Agradecimiento}

El presente artículo fue redactado gracias a una ayuda del Ministerio de Cultura de República Checa, en el marco del proyecto Temap, del programa NAKI.

\section{Bibliografía y referencias}

ALA. Magirt. http://www.ala.org/magirt

ALA (2013). Magirt. Training \& presentations.

http://magirt.ala.libguides.com/trainingsandpresentations

ALA (2010). RDA: Resource Description and Access. Chicago: American Library Association. ISBN: 9780838910931

ALA. RDA Toolkit.

http://access.rdatoolkit.org

ALA (2013). RDA Toolkit. D.2. MARC 21 format for bibliographic data mapped to RDA elements.

http://access.rdatoolkit.org/document.php?id=jscmap2

ALA (2013). RDA: back to the basics. An Alcts pre-conference. June 28. Chicago: ALA.

ALA (2010). RDA: resource description and access. Chicago: American Library Association. ISBN: 9780838910931

ALA. RDA in translation.

http://www.rdatoolkit.org/translation

Anglo-americká katalogizační pravidla: 2. vydání: revize 1988. Praha: Národní knihovna ČR, 1994-. ISBN: 8070501871

Andrew, Paige G. RDA and cartographic materials: mapping a new route. RDA webinar. ALA, 2011.

http://www.ala.org/alcts/confevents/upcoming/webinar/ cat/092811

Andrew, Paige G. RDA and cartographic materials: mapping a new route, an Alcts webinar. Questions \& answers log. RDA webinar. ALA, 2011.

http://alcts.ala.org/ce/0928_2011_RDA_and_Cartographic_ Materials_QA.doc

Andrew, Paige G.; Laarsgard, Mary-Lynette (2014). RDA and cartographic resources, Chicago: ALA Editions, 2014. ISBN: 9780838911310

Andrew, Paige G.; Moore, Susan. Maps the RDA way. Presentation at ALA meeting 2013. Chicago: Magirt, 2013. http://lgdata.s3-website-us-east-1.amazonaws.com/ docs/3001/802199/MAGIRT_2013_Program_Presentation_ Slides.pdf

Andrew, Paige G.; Moore, Susan. Maps the RDA way-the sequel. Webinar.

http://ala.adobeconnect.com/p658auoia2i 
Celbová, Ludmila (2001). Funkční požadavky na bibliografické záznamy: závěrečná zpráva. Praha: Národní knihovna ČR. ISBN: 8070504005

http://www.ifla.org/files/assets/cataloguing/frbr/frbr-cs. $p d f$

IFLA. Ustanovení mezinárodních principů katalogizace. http://www.ifla.org/files/assets/cataloguing/icp/icp_2009cs.pdf

IFLA Study Group on the Functional Requirements for Bibliographic Records. Final report. München: K.G. Saur, 1998. http://archive.ifla.org/VII/s13/frbr/frbr.pdf

IFLA Working Group on Functional Requirements and Numbering of Authority Records (Franar). Functional requirements for authority data: a conceptual model. Ed. by Glenn E. Patton. München: K.G. Saur, 2009.

International standard bibliographic description (ISBD). München: K. G. Saur, 2007.

Joint Steering Committee for Development of RDA. RDA: Resource description and access.

http://www.rda-jsc.org/rda.html

Joint Steering Committee for Development of RDA. RDAFRAD mapping.

http://www.rda-jsc.org/docs/5rda-rdafradmappingrev.pdf

Kárászová, Lenka (2009). Vývoj katalogizačních pravidel "Resource description and access". Praha: Univerzita Karlova v Praze.

Kiorgaard, Deirdre. RDA to FRBR mapping. http://www.rda-jsc.org/docs/5rda-rdafrbrmappingrev2.pdf

Kuhagen, Judy A. (2011). RDA LC reference staff briefing. $h t t p: / / w w w . r d a-j s c . o r g / d o c s / 10_{-} 11_{-} 30_{-}$ LCReferencestaffbriefingrev2011.ppt

Library of Congress. Accessing policy statements. http://www.loc.gov/aba/rda/lcps_access.html

Library of Congress. 264 - Production, publication, distribution, manufacture and copyright notice. http://www.loc.gov/marc/bibliographic/bd264.htm/

Library of Congress. RDA core elements.

http://www.loc.gov/aba/rda/pdf/core_elements.pdf

MARC 21: bibliografický formát. Praha: Národní knihovna České republiky, 2003. ISBN: 8070504277

MARC 21: formát pro autority. Praha: Národní knihovna ČR, 2004. ISBN: 8070504412

Mendes, Luiz (2013). Access point changes: with exercises. In: RDA: Back to the basics. An Alcts pre-conference. Presentation at ALA meeting. Chicago: ALA.

Moore, Susan (2013). About RDA cataloguing (email).

Moore, Susan (2013). Natural scale indicator. May, 31st. https://www.youtube.com/watch?v=vSus_5bt440

OCLC. OCLC RDA policy statement.

http://www.oclc.org/en-US/rda/new-policy.htmI

OCLC. Bibliographic formats and standards.

http://www.oclc.org/bibformats/en.html

Oliver, Chris (2013a). RDA: highlights of descriptive changes. In: RDA: Back to the basics. An Alcts pre-conference. Presentation at ALA meeting. Chicago: ALA.

Oliver, Chris (2013b). Understanding RDA: A guide to some basic concepts. In: RDA: Back to the basics. An Alcts pre-conference. Presentation at ALA meeting. Chicago: ALA.

Rankin, Kate; Larsgaard, Mary-Lynette (2012). RDA for cartographic resources. Hawaii: University of Hawaii at Manoa, 2012. Presentation at WAML meeting.

http://digitalscholarship.unlv.edu/libfacpresentation/98

Welsh, Anne; Batley, Sue (2012). Practical cataloguing: $A A C R, R D A$ and MARC21. Chicago: Neal-Schuman Publishers, imprint of American Library Association. ISBN: 9781 555707439

Whitacre, Cynthia; Fujikawa, Georgia (2013). View from utilities. In: RDA: back to the basics. An Alcts pre-conference. Presentation at ALA meeting 2013. Chicago: ALA.

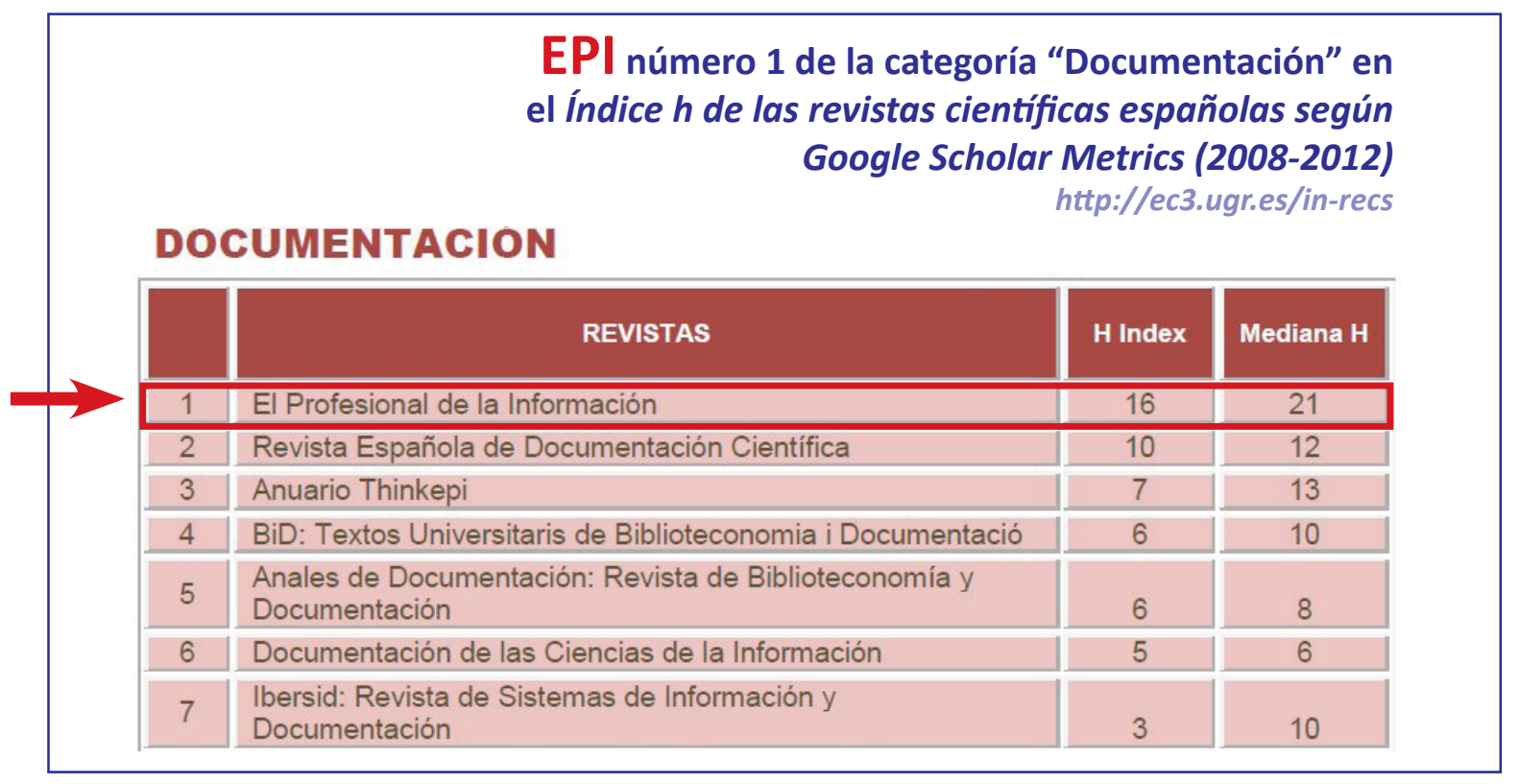

